were hypertrophied; while there was also suppuration in the right kidney, dilated ureters, and pyelitis.

The object of Dr. Ogle's paper, "On the Influence of the Cervical Portions of the Sympathetic Nerve and Spinal Cord upon the Eye and its Appendages," is to apply the results of various experiments-showing the influence possessed by the sympathetic in the neck and the upper part of the spinal cord upon the iris and upper eyelid--to the advancement of clinical medicine. The curtain of the iris not only contains two sets of muscular fibres (a circular set by which the pupil is contracted, and a radiating set by which it is enlarged), but is also under the domination of two separate and distinct sources of innervation. The third cranial nerve is found to control the circular or contracting fibres; while the sympathetic, by virtue of communications with the lenticular ganglion, is found to control the dilator or radiating fibres. Consequently, if the influence of the third pair be destroyed, the pupil becomes dilated, inasmuch as the dilator-fibres-those presided over by the sympathetic-are unopposed; while, if the influence of the third cranial pair be left unimpaired, and that of the sympathetic be destroyed by section or extreme pressure, then the pupil becomes contracted. Comments are then made upon the history of the various experiments upon which these statements are founded, and also upon those from which it is concluded that in certain parts of the spinal cord resides the power or influence which acts upon the dilator-fibres of the iris passing to that structure through the sympathetic via the roots of certain cervical and dorsal nerves. From these latter it is apparent that the same paralysis of the dilator-fibres of the iris which follows section of the sympathetic in the neck, follows also the severance of such fibres as connect the sympathetic with the spinal cord, as also the section or destruction of the spinal cord itself in certain parts. Hence it can be imagined, that any cause of extreme pressure acting upon the various portions of the nervous system alluded to would cause a contracted state of the pupil on the side corresponding to that on which the extreme pressure existed. In this way Dr. Gairdner, of Edinburgh, has attempted to explain those cases in which a contracted state of the pupil has coincided with an intrathoracic aneurism. Examples are adduced in which pressure from aneurism upon the sympathetic in the neck had produced a contracted pupil. Then follow instances in which extreme pressure from other causes than aneurism produced a like effect upon the pupil, as in the case of enlarged glands, malignant tumours, \&c.; and these are succeeded by several cases in which a contracted pupil had been observed in injuries of the spinal cord itself. The occurrence of dilatation of the pupil, in certain cases of aneurism, is then attempted to be explained; and the paper concludes with some remarks on ptosis and convergent strabismus, so far as their occurrence has been noticed in connexion with pressure about the neck from aneurism or other sources.

A case of premature puberty, related by Mr. Smart, forms a valuable paper. The subject of it was born in Manchester, in January, 1853; and at the age of three years and a half had an appearance of hair upon the pubes, followed by a menstrual discharge. Though out of health before the first occurrence of the catamenia, she has since become strong and well, the menses recurring with perfect regularity up to the time of observation (May, 1857), at which period she was three feet seven inches in height, and weighed fifty-two pounds. A table of twelve other recorded examples of premature puberty is appended.

The chief remaining papers, although possessing much merit and general value, must be dismissed in a few remarks. Dr. $R$. Lee has an essay with a supplement, having for its object the demonstration of the existence of a decidua around the ovum in cases of tubal gestation. Dr. Marcet relates an easy and very practical method for analyzing the fæces in certain morbid conditions; and shows that in three instances of disease, where the bile was prevented from flowing into the duodenum, the fæces yielded a quantity of crystallizable fatty acids (margaric and stearic acids), which, except in cases depending upon peculiar diet, do not occur in healthy evacuations as immediate results. Mr. Coulson relates the clinical history of a young woman who had an ulcer leading into a cavity in the tibia, which was lined by a white glistening membrane; and from which a considerable number of hydatids were removed. The report is valuable, because hydatids are very rarely wet with in the osseous system; though it is remarkable that in the majority of published cases the tibia has been the bone affected. Dr. H. R. Silvester gives a contribution to the science of teratology by the notes of a case of congenital deformity in both upper extremities, and deductions therefrom. The ophthalmoscopic examination of many instances of glancoma, and the dissection of several eyes affected with this disease, have furnished to Mr. Hulke some valuable facts, which he details. Dr. Fox's observations on the pathology of the glandular structures of the stomach contain the result of a series of microscopical examinations of one hundred stomachs, taken indiscriminately from the bodies brought for post-mortem examination to the Pathological Institute of the Charite Hospital, Berlin, under the direction of Professor Tirchow. And, lastly, Mr. Charles Hawkins gives an account of a case of vesical calculus, in which the stone was successfully removed by lithotrity; the point of interest being this -that there existed a communication between the bladder and the intestine.

On the Restoration of Motion by Forcible Extension and Rup. ture of the Uniting Medium of Partially-Anchylosed Surfaces. By Bervard E. BrodHcrst, Senior Assistant-Surgeon to the Royal Orthopedic Hospital, \&c. pp. 39. London: Adlard. 1858.

THE author has treated thirty-two cases of partially-anchylosed joints by rupture of the uniting medium and forcible ex. tension, with the following results:-In eleven instances, complete power of motion was gained; in fourteen, partial but useful motion was restored; and, in seven, the limb was rendered straight, and the joint has remained stiff. He divides these cases into two classes: first, those in which, from great muscular contraction, it is necessary to divide tendons and fascire, and subsequently to rupture the adhesions; and, secondly, those in which muscular contraction may be sufficiently overcome by the exhibition of chloroform to enable the adhesions to be ruptured without the use of the knife. The author most judiciously insists, that no further motion nor examination of the joint should be permitted after the adhesions have been ruptured. His observations, on this important subject, may be read with advantage.

\section{DETECTION OF CALLUS IN UNUNITED FRACTURES.}

To the Editor of THE LAYCET.

Sir, -- On the 15th of October last I was called to see a boy who had fallen out of a cart. When I arrived, I found he had a fracture of the lower third of the left femur. With the assistance of the boy's father, I first rolled the whole limb from the toes upwards, and then with two long splints, on either side, one extending from the arm-pit to the foot, the other placed on the inside of the limb-all carefully and evenly rolled, and the whole limb kept from moving by a sand-bag; and within one day short of three veeks the fracture was united, so firmly and so evenly that it would have defied the most skil. ful surgeon to tell where the bone had been broken.

If the foregoing case is of any service in assisting to show the gross ignorance and disgraceful conduct of those persons who gave evidence against Mr. Wollaston, please let it be in. serted in the next number of your journal.

$$
\text { J. G. Sproston, M.R.C.S. }
$$

Marshfield, near Chippenham, January, 185.

University Colleae Hospital.-John Hibbert, Esq., of Braywick Lodge, Maidenhead, has made another munificent donation of $£ 100$, being the fifth of the same amount, to the invested funds of this hospital. 